\title{
Beam loading compensation of traveling wave linacs through the time dependence of the rf drive
}

\author{
Nathan Towne* \\ 1094 White Oak Lane, Farmington, New York 14425, USA \\ James Rose ${ }^{\dagger}$ \\ National Synchrotron Light Source II, Brookhaven National Laboratory, Upton, New York 11973-5000, USA
}

(Received 6 January 2011; published 30 September 2011)

\begin{abstract}
Beam loading in traveling-wave linear accelerating structures leads to unacceptable spread of particle energies across an extended train of bunched particles due to beam-induced field and dispersion. Methods for modulating the rf power driving linacs are effective at reducing energy spread, but for general linacs do not have a clear analytic foundation. We report here methods for calculating how to modulate the rf drive in arbitrarily nonuniform traveling-wave linacs within the convective-transport (power-diffusion) model that results in no additional energy spread due to beam loading (but not dispersion). Varying group velocity, loss factor, and cell quality factor within a structure, and nonzero particle velocity, are handled.

DOI: 10.1103/PhysRevSTAB.14.090402

PACS numbers: $41.75 . \mathrm{Ht}$
\end{abstract}

\section{INTRODUCTION}

Beam loading in traveling-wave linear accelerators results in the variation (decline) of the energy gain of particles in a train passing through the structure. With constant rf drive and beam current, this decline terminates after the fill time of the structure following the start of injection. Thus, if injection exceeds the fill time, particles injected after the fill time see the same energy gain. There is no energy spread among those particles. But when the duration of injection is shorter than or comparable to the fill time, energy spread cannot be avoided via this route. A number of methods are available that reduce the variation of energy gain in this latter regime. One in particular ramps the injected rf amplitude and phase while the linac fills with $\mathrm{rf}$ power in such a way that the variation of energy gain of beam subsequently injected is greatly reduced ([1], Sec. 4.2.9, and [2]). This technique is complicated by details such as klystron saturation, temperature variations in the structure, nonzero beam transit time, and dispersion [3], and is naturally implemented adaptively $[4,5]$.

The power-diffusion model $[6,7]$ has been a context within which to estimate linac behavior and performance. This model is a convective-transport (fluid-dynamical) model of traveling-wave linacs in which energy moves through these structures with a position-dependent speed determined by the dimensions of the irises loading the waveguide making up these structures. This model is

\footnotetext{
*towne56@uchicago.edu

†rose@bnl.gov
}

Published by the American Physical Society under the terms of the Creative Commons Attribution 3.0 License. Further distribution of this work must maintain attribution to the author(s) and the published article's title, journal citation, and DOI. described in detail in Sec. II to provide crucial context for later sections.

Note that the convective-transport model is a spatialcontinuum approximation of the full dynamics of traveling-wave structures. Given that these iris-loaded waveguide structures consist of high quality factor $(Q)$ resonators with weak coupling between them, they are grainy on the scale of the cells. While a continuum approximation is useful for spatial variation of fields that is slow compared to the cell size, it inevitably fails at sufficiently fine spatial (and temporal) scales. More detailed electrical $[8,9]$ or other [10] models are required to describe such behavior [3]. In fact, in linacs with relatively few cells, continuum models may not be useful at all. But where there are many cells, a continuum model has the ability to usefully model long-wavelength behavior. In any case, the effects of dispersion are beyond the scope of this paper and will not be discussed further. The reader may consult the references and other literature where these effects have more recently been studied extensively. The inevitable presence of machine instabilities and dispersion mean that the results of this paper do not obviate the need for adaptive feedforward in real linacs.

In 1963 Chu calculated within the convective-transport model how to ramp the rf to prepare the static beam-loaded field profile in the linac, which results in constant energy gain for beam subsequently injected [11]. Chu's treatment was limited to constant-gradient linacs with constant impedance along its length. This paper extends Chu's work to arbitrarily varying traveling-wave linacs, and does so using simpler mathematical methods. Instead of the use of a Green's function, it exploits the fluid-dynamical nature of the partial differential equations describing the flow of energy and field within these structures. The problem is reduced to two ordinary differential equations, the first 
describing the properties of the flow, and the second describing the variation of field comoving with the flow. These more general results are used to calculate the power ramp that prepares the static beam-loaded field profile prior to injection. The general result is given in Sec. III.

This result has a simpler form for linacs engineered for constant gradients (Sec. IV). In these linacs, the latter differential equation describes, without beam loading, unchanging fields as they move with the flow. This simplification allows equating the static field at a particular point along the linac to the field at the entrance to the linac at an earlier time determined by the group velocity, thus determining the corresponding driving field at the entrance to the linac. Further simplifications result from constant loss factor (Sec. IVA), and constant loss factor and constant cell quality factor (Sec. IV B). In the latter case, the analytic result is that $\mathrm{rf}$ is ramped linearly in amplitude for constant energy, and that the slope of the ramp is proportional to current in the pulse, a result already known to provide a high degree of compensation $[4,12]$ when the slope of the ramp has the correct value. This analytic result is applied numerically to illustrate it.

\section{CONVECTIVE-TRANSPORT MODEL OF TRAVELING-WAVE LINACS}

The cells of traveling-wave linacs considered here have uniform length (constant-beta linacs) but whose coupling, quality factors, and impedances (or loss factors) may vary. While the cell length is a fundamental length scale in these structures, on larger scales a continuous approximation proves useful. On these scales rf energy density $U(z, t)$ moves with group velocity $v_{g}(z)$ and has power flow $P(z, t)$.

There are two fundamental equations needed to describe the behavior of these quantities, the first for energy accounting,

$$
\frac{\partial}{\partial t} U(z, t)+\frac{\partial}{\partial z} P(z, t)=-\frac{\omega}{Q} U(z, t)-\operatorname{Re}\left[\hat{E}_{z}(z, t)^{*} \hat{i}(z, t)\right]
$$

where $\omega$ is the $\mathrm{rf}$ frequency, $Q$ is the possibly $z$-dependent quality factor of the cells of the structure, $\hat{i}$ is the beam current, and $\hat{E}_{z}$ is the electric field averaged over cells including the transit-time factor. The left-hand side (lhs) is an equation of continuity for energy conservation, and on the right-hand side (rhs) are source and sink terms, the first for wall dissipation, and the second for beam loading.

The second equation is the relation between energy density and the unidirectional flow of power:

$$
P(z, t)=v_{g}(z) U(z, t)
$$

where $v_{g}$ is the group velocity of the structure. Combining these equations, we have

$$
\frac{\partial}{\partial t} U+v_{g} \frac{\partial}{\partial z} U=-\left(v_{g}^{\prime}+\frac{\omega}{Q}\right) U-\operatorname{Re}\left(\hat{E}_{z}^{*} \hat{i}\right) .
$$

In this equation, the $z$ and $t$ dependence of $U, \hat{E}_{z}$, and $\hat{i}$, and the $z$ dependence of $v_{g}, k$, and $Q$, are suppressed. The prime indicates differentiation with respect to $z$. The lhs of this equation is the convective derivative $D / D t$ of $U$ in the velocity field $v_{g}$ :

$$
D_{t} \equiv \frac{D}{D t} \equiv \frac{\partial}{\partial t}+v_{g} \frac{\partial}{\partial z}
$$

which is the derivative comoving with the flow $v_{g}$. In other fluid-dynamical contexts, the velocity field $v_{g}$ can depend on both space and time and the $z$ derivative is the multidimensional gradient; here, however, $v_{g}$ is tied to the one-dimensional accelerating structure and so is not time dependent, and the spatial derivative is of one variable. So Eq. (3) tracks the gain and loss of the energy density $U$ as $U$ moves as a fluid through the structure. One can see that, due to the $v_{g}^{\prime}$ term, at least in the absence of sources and sinks, energy tends to pile up where the flow velocity is low, and tends to thin out where it is high.

Boundary/initial conditions for Eq. (3) exist at $t=0$ and $z=0$. Energy density at time $t=0$ is the prescribed starting condition of the linac-usually zero. The energy density at the $z=0$ boundary is density launched into the linac by the rf power applied to the first cell.

The complex-valued electric field $\hat{E}_{z}$ is related to $U$ through

$$
\left|\hat{E}_{z}\right|^{2} / 4 k=U,
$$

where $k=\omega / 2 Q \times r$ is the loss factor per unit length of the accelerating mode of the structure, and $r$ is the impedance. Equation (5) does not determine the phase of $\hat{E}_{z}$, which will later be determined by another criterion. The convective equation for $\hat{E}_{z}$ is obtained by first dividing Eq. (3) by $U$ :

$$
0=\frac{D_{t} \hat{E}_{z}+2 k \hat{i}}{\hat{E}_{z}}+\Gamma+\text { c.c. }
$$

where c.c. means the complex conjugate, and the $z$-dependent damping rate $\Gamma$ is

$$
\Gamma(z)=\frac{1}{2}\left(v_{g}(z)^{\prime}-v_{g}(z) \frac{k(z)^{\prime}}{k(z)}+\frac{\omega}{Q(z)}\right)
$$

corresponding to the $\alpha v_{g}$ term of Eq. (1) of Ref. [11]. As will be seen below, $\Gamma$ is a physical damping rate of field as it moves with the flow. Given that the two terms of Eq. (6) are complex conjugates of each other and that their sum is zero, they are each a pure imaginary function of $z$ and $t$, namely $\pm j f$, where $f$ is real valued and is unconstrained by Eq. (6). The function $f$ is a function of the linac structure and, because the linac is static, it cannot depend on time. The equation for $\hat{E}_{z}$ is then

$$
\frac{D_{t} \hat{E}_{z}+2 k \hat{i}}{\hat{E}_{z}}=-\Gamma(z)+j f(z) .
$$


The slope function $f$ corresponds to $\beta v_{g}$ of Eq. (1) of Ref. [11].

In the absence of beam, $\hat{E}_{z}$ evolves as it moves with the flow as

$$
\hat{E}_{z}[z, t(z)]=\hat{E}_{z}[0, t(0)] \times e^{-\alpha(z)+j \varphi(z)},
$$

where $\alpha$ and $\varphi$ are definite integrals of the two terms of Eq. (8) describing damping and phase, respectively,

$$
\begin{aligned}
& \alpha(z)=\int_{0}^{z} \frac{d z}{v_{g}(z)} \Gamma(z) \\
& \varphi(z)=\int_{0}^{z} \frac{d z}{v_{g}(z)} f(z) .
\end{aligned}
$$

The quantity $\alpha$ is a $z$-dependent voltage attenuation coefficient that describes how the magnitude of a slice of $\hat{E}_{z}$ changes as it moves through the structure (without beam loading). For example, in a constant-gradient structure, it is identically zero, as is $\Gamma$. The phase shift $\varphi$ contains the linear phase advance, which for acceleration must match the phase advance of $\hat{i}$. It can in principle contain arbitrary nonlinear terms causing phase slip, but in this paper those terms are all assumed tuned away during the engineering of the structure. Furthermore, without loss of generality in this model, the linear phase advance is here assumed to be zero. So $f(z)=0$ and the time-dependent equation for $\hat{E}_{z}$ is

$$
D_{t} \hat{E}_{z}(z, t)=-\Gamma(z) \hat{E}_{z}(z, t)-2 k(z) \hat{i}(z, t),
$$

showing how $\Gamma$ is a comoving damping rate. As in Eq. (3) for $U$, there are initial/boundary conditions, one being the field along the linac at some reference time, and the second being the field $\hat{E}_{z}(z=0, t)$ applied to the upstream end of the linac by incoming rf power, which is then carried through the linac by the flow.

The time-independent (static) equation is

$$
\frac{d}{d z} \bar{E}_{z}(z)=-\frac{\Gamma(z)}{v_{g}(z)} \bar{E}_{z}(z)-2 \frac{k(z)}{v_{g}(z)} \hat{i}(z)
$$

The initial condition $\bar{E}_{z}(z=0)$ may have any phase and magnitude.

In general, the phases of $\hat{E}_{z}$ and $\bar{E}_{z}$ vary with $z$, and $t$ in the case of $\hat{E}_{z}$. But when the fixed phase of the rf drive is the same as that of $\hat{i}$, the phases of $\hat{E}_{z}$ and $\bar{E}_{z}$ remain fixed at that of $\hat{i}$ and the beam is said to be on crest.

In general, the linac's fill time $t_{\text {fill }}$ is

$$
t_{\text {fill }}=\int_{0}^{l} \frac{d z}{v_{g}(z)},
$$

where $l$ is the length of the linac, and the time for the field at $z=0$ to be carried by the flow to $z$ is

$$
t_{\mathrm{f}}(z)=\int_{0}^{z} \frac{d z^{\prime}}{v_{g}\left(z^{\prime}\right)} \text {. }
$$

This model is often referred to as the power-diffusion model, but the movement of energy among cells is essentially convective and does not involve diffusion. For this reason, the core of this model is instead fluid flow. This distinction and the transit times of Eqs. (14) and (15) are central to this treatment of beam loading in these linacs.

\section{CONSTANT ENERGY GAIN IN TRAVELING-WAVE LINACS}

The problem is to prepare a linac, whose field satisfies the time-dependent Eq. (12) without beam loading,

$$
D_{t} \hat{E}_{z}(z, t)=-\Gamma(z) \hat{E}_{z}(z, t),
$$

with field $\hat{E}_{z}(z=0, t)$ at its entrance so that at the end of the fill

$$
\hat{E}_{z}\left(z, t_{\text {fill }}\right)=\bar{E}_{z}(z)
$$

where $\bar{E}_{z}(z)$ satisfies the static (beam-loaded) equation (13). Were beam to be switched on everywhere in the linac at this time, the static equation (13) would be satisfied for as long as constant beam and rf remain on.

First a note about the beam transit time. Group velocities in traveling-wave linacs are typically one-to-ten percent of the speed of light. This is fairly small, particularly on the low end of that range, and useful approximations with relativistic particles may be had by assuming that the current $\hat{i}$ is switched on simultaneously everywhere in the linac. But significant error is inevitably acquired via that approximation. For this reason, the approximation is not applied here even though there is some additional complexity added to the derivation. In what follows, injection starts at $t_{\text {inj }}=t_{\text {fill }}-l / v_{b}$, where $v_{b}$ is the particle velocity. Between times $t_{\text {inj }}$ and $t_{\text {fill }}$, rf power is advancing in the structure as beam is also advancing. The ramp is timed so that at the time beam arrives at a point $z_{0}$ in the linac, $\mathrm{rf}$ field of intensity $\bar{E}_{z}\left(z_{0}\right)$ has simultaneously arrived. In this derivation, particle velocity is assumed constant during acceleration through the linac section, although departures from this condition do not fundamentally change the derivation or conclusions.

Figure 1 is a space/time plot of the linac for $0 \leq t \leq t_{\text {fill }}$ and $0 \leq z \leq l$. The leading particles progress through the structure on the diagonal dashed line, and the structure is filled with both beam and rf power at time $t_{\text {fill }}$. The shaded region is linac under beam loading, while the unshaded region evolves without beam. The static field $\bar{E}_{z}$ is established on the dashed boundary between them (and later) through the rf drive $\hat{E}_{z}(z=0, t)$ during $0 \leq t \leq t_{\text {inj }}$ to be determined in this section.

The idea is to convert the partial differential equation (16) to an ordinary differential equation parametrized by $z_{0}$ representing a point in the linac. This differential equation is then integrated backward from the $z_{0}$-dependent time $t=t_{\text {fill }}-\left(l-z_{0}\right) / v_{b}$ (the time of first arrival of beam at 


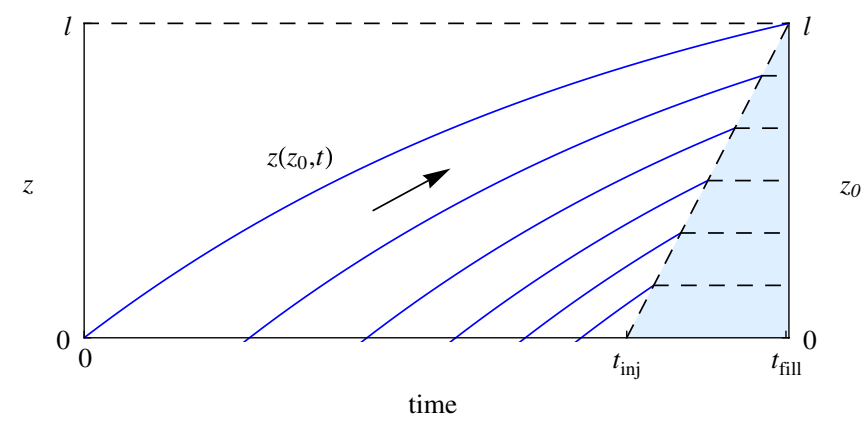

FIG. 1. The flow of the vector field $v_{g} \partial_{z}$ for the constant- $k$ constant- $Q$ linac of Sec. IV B. The curves correspond to six values of $z_{0}$ with $z\left[z_{0}, t_{\text {fill }}-\left(l-z_{0}\right) / v_{b}\right]=z_{0}$. The start of

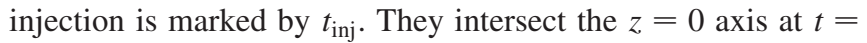
$t_{\text {fill }}-t_{\mathrm{f}}\left(z_{0}\right)$. The shaded region shows the region and duration of beam loading during injection. During that time the static field $\bar{E}_{z}(z)$ is established. The beam transit time $t_{\text {fill }}-t_{\text {inj }}=l / v_{b}$ is shown exaggerated.

point $z_{0}$ ), with $z$ depending on $t$ through Eq. (15), to location $z=0$ to determine the applied field $\hat{E}_{z}(z=0, t)$ that fills the linac to $\bar{E}_{z}$ at time $t_{\text {fill }}-\left(l-z_{0}\right) / v_{b}$. In this scheme, the static field $\bar{E}_{z}$ is established earlier than is given in Eq. (17), which is modified to

$$
\hat{E}_{z}\left[z_{0}, t_{\text {fill }}-\left(l-z_{0}\right) / v_{b}\right]=\bar{E}_{z}\left(z_{0}\right) .
$$

Preparation of the static field $\bar{E}_{z}$ is a two-step process: the first to construct the flow of the vector field $v_{g} \partial_{z}$ ([13], Sec. 3.5), and the second to integrate the ordinary differential equation corresponding to Eq. (16) backward in time. The idea of a flow is a unifying concept applied here; even though the manifold is almost trivially one dimensional, some of the language is nevertheless retained.

We begin with the construction of the flow. The flow of the vector field $v_{g} \partial_{z}$ is defined to be the function $z\left(z_{0}, t\right)$ with

$$
\frac{d}{d t} z\left(z_{0}, t\right)=v_{g}\left[z\left(z_{0}, t\right)\right]
$$

having the initial condition

$$
z\left[z_{0}, t=t_{\text {fill }}-\left(l-z_{0}\right) / v_{b}\right]=z_{0}
$$

for each $0 \leq z_{0} \leq l$. The $z=0$ intercept is

$$
z\left[z_{0}, t_{\text {fill }}-\left(l-z_{0}\right) / v_{b}-t_{\mathrm{f}}\left(z_{0}\right)\right]=0 .
$$

To illustrate, the flow of the linac of Sec. IV B is shown in Fig. 1. Each line is for a single value of $z_{0}$ (axis on the right).

With $z=z\left(z_{0}, t\right)$ defined by Eq. (19) in this way, the convective derivative $D / D t$ in Eq. (16) becomes the ordinary derivative in the equation

$$
\frac{d}{d t} \hat{E}_{z}\left[z\left(z_{0}, t\right), t\right]=-\Gamma\left[z\left(z_{0}, t\right)\right] \hat{E}_{z}\left[z\left(z_{0}, t\right), t\right]
$$

Next, we divide by $\hat{E}_{z}\left[z\left(z_{0}, t\right), t\right]$ and integrate from $t_{\text {fill }}-$ $\left(l-z_{0}\right) / v_{b}-t_{\mathrm{f}}\left(z_{0}\right)$ to $t_{\text {fill }}-\left(l-z_{0}\right) / v_{b}$ where the flow advances from $z=0$ to $z=z_{0}$, with the result

$$
\log \frac{\hat{E}_{z}\left[z_{0}, t_{\text {fill }}-\left(l-z_{0}\right) / v_{b}\right]}{\hat{E}_{z}\left[z=0, t_{\text {fill }}-\left(l-z_{0}\right) / v_{b}-t_{\mathrm{f}}\left(z_{0}\right)\right]}=-\alpha\left(z_{0}\right),
$$

where $\alpha$ is defined by Eq. (10). By Eq. (18), the numerator of the lhs is $\bar{E}_{z}\left(z_{0}\right)$. Changing variable

$$
t_{\text {fill }}-\left(l-z_{0}\right) / v_{b}-t_{\mathrm{f}}\left(z_{0}\right) \rightarrow t
$$

and inverting Eq. (24) for $z_{0}=z_{0}(t)$, we get the expression for the field at the entrance of the linac that prepares $\hat{E}_{z}$ to the static $\bar{E}_{z}$ :

$$
\hat{E}_{z}(0, t)=\bar{E}_{z}\left[z_{0}(t)\right] \times e^{\alpha\left[z_{0}(t)\right]} .
$$

Equations (5) and (2) are then used to determine the delivered (forward minus reflected) rf power needed to launch $\hat{E}_{z}(0, t)$ at the entrance to the linac.

\section{CONSTANT-GRADIENT LINACS}

The condition for a constant-gradient structure in the absence of beam loading is that the damping rate $\Gamma$ in Eq. (7) is zero along the length of the linac. The time-dependent equation without beam loading Eq. (12) becomes

$$
D_{t} \hat{E}_{z}=0
$$

or that the field does not change as it moves with the flow. The time-independent Eq. (13) with beam loading is

$$
\frac{d}{d z} \bar{E}_{z}(z)=-2 \frac{k(z)}{v_{g}(z)} \hat{i}
$$

which integrates to

$$
\bar{E}_{z}(z)=\bar{E}_{z}(0)-2 \hat{i} \int_{0}^{z} d z^{\prime} \frac{k\left(z^{\prime}\right)}{v_{g}\left(z^{\prime}\right)} .
$$

$\Gamma=0$ in Eq. (7) can be integrated for $v_{g}(z):$

$$
v_{g}(z)=\frac{k(z)}{k(0)} v_{g}(0)-k(z) \int_{0}^{z} d z^{\prime} \frac{\omega}{k\left(z^{\prime}\right) Q\left(z^{\prime}\right)} .
$$

Because $\alpha$ in Eq. (25) is zero, the constant energy gain equation (25) reduces to the simpler

$$
\hat{E}_{z}(0, t)=\bar{E}_{z}\left[z_{0}(t)\right]
$$

or that $\bar{E}_{z}\left(z_{0}\right)$ at time $t=t_{\text {fill }}-\left(l-z_{0}\right) / v_{b}$ is prepared by launching that same rf field at time $t=t_{\text {fill }}-\left(l-z_{0}\right) /$ $v_{b}-t_{\mathrm{f}}\left(z_{0}\right)$ at the linac entrance $(z=0)$. Further simplifications result from assumptions about $k$ and $Q$.

\section{A. Uniform (constant) loss factor $k$}

When the loss factor $k$ is constant, Eq. (28) for the static field integrates to 


$$
\bar{E}_{z}(z)=\bar{E}_{z}(0)-2 k \hat{i} \times t_{\mathrm{f}}(z)
$$

because, physically, beam drags down the rf field at a constant rate. Equation (30) and (31) give us the rf field at the entrance for constant energy gain:

$$
\hat{E}_{z}(0, t)=\bar{E}_{z}(0)-2 k \hat{i} \times\left(t_{\text {fill }}-\frac{l-z_{0}(t)}{v_{b}}-t\right) .
$$

Thus, for constant loss factor $k$ and particle velocity $v_{b}$ large compared to the group velocity $v_{g}$, an approximately linear ramp with slope $2 k \hat{i}$ prepares $\bar{E}_{z}(z)$. From Eq. (29), the group velocity $v_{g}(z)$ is

$$
v_{g}(z)=v_{g}(0)-\int_{0}^{z} d z^{\prime} \frac{\omega}{Q\left(z^{\prime}\right)} .
$$

\section{B. Uniform (constant) loss factor and $Q$}

If both the loss factor $k$ and $Q$ are constant in a constantgradient linac, further simplifications occur. First, the group velocity $v_{g}(z)$ has the well-known constant slope matched to the $Q$ :

$$
v_{g}(z)=v_{g}(0)-\frac{\omega}{Q} \times z .
$$

Equation (15) for the transit time integrates simply to

$$
t_{\mathrm{f}}(z)=-\frac{Q}{\omega} \log \frac{v_{g}(z)}{v_{g}(0)} .
$$

Energy gain as a function of time during the fill with constant input power, without beam loading, and with the zero-transit-time approximation has a particularly simple form. Assume $\bar{E}_{z}, E_{z}=\hat{E}_{z}$, and $i=\hat{i}$ are all real (on crest). Then energy gain as a function of filled length $z$ is

$$
\Delta E=q E_{z} \times z,
$$

where $q$ is the particle charge, while the time to fill that length is

$$
t_{\mathrm{f}}(z)=-\frac{Q}{\omega} \log \left(1-\frac{\omega}{v_{g}(0) Q} z\right)
$$

from Eqs. (35) and (34). Inverting Eq. (37) for $z$ as a function of $t$ and inserting into Eq. (36) gives us the energy gain as a function of time during the fill,

$$
\Delta E(t)=q E_{z} v_{g}(0) \frac{Q}{\omega}\left(1-e^{-\omega t / Q}\right) .
$$

This is an exponential having a time constant $\tau=Q / \omega$, which is half the linac cells' damping time.

The static beam-loaded voltage $\int d z \bar{E}_{z}$ seen by the particles is expressible in closed form. Starting with Eqs. (31), (34), and (35),
TABLE I. Parameters of the linac model shown in the figures.

\begin{tabular}{lrcc}
\hline \hline Parameter & Symbol & Value & Unit \\
\hline Length & $l$ & $=5.2$ & $\mathrm{~m}$ \\
Frequency & $\omega / 2 \pi$ & $=3$ & $\mathrm{GHz}$ \\
Quality factor & $Q$ & $=14137$ & \\
Group velocity & $v_{g}$ & $=0.033 \rightarrow 0.01$ & $\mathrm{c}$ \\
Impedance & $r$ & $=43 / 2$ & $\mathrm{M} \Omega / \mathrm{m}$ \\
Loss factor & $k$ & $=28.7 / 2$ & $\mathrm{~V} / \mathrm{pC} \mathrm{m}$ \\
Time constant & $Q / \omega$ & $=750$ & $\mathrm{~ns}$ \\
Fill time & $t_{\text {fill }}$ & $=904$ & $\mathrm{~ns}$ \\
Particle velocity & $v_{b}$ & $=\infty$ & \\
\hline \hline
\end{tabular}

$$
\begin{aligned}
V & =l \bar{E}_{z}(0)-2 k i \int_{0}^{l} d z t_{\mathrm{f}}(z) \\
& =l \bar{E}_{z}(0)-2 k i \frac{Q}{\omega}\left(l+\frac{Q v_{g}(l)}{\omega} \log \frac{v_{g}(l)}{v_{g}(0)}\right)
\end{aligned}
$$

where $\bar{E}_{z}(0)$ sets the energy gain.

As in Sec. IVA, Eq. (32) prescribes the ramp of $\hat{E}_{z}$ for constant beam-loaded energy gain, and Eqs. (5) and (2) relate that field to delivered power. Equation (32) does not presume that $Q$ is constant. Also, the result from Sec. IVA that for particle velocity large compared to the group velocity a linear ramp of $\hat{E}_{z}(0, t)$ prepares $\bar{E}_{z}$ carries over to this section.

These theoretical results are numerically illustrated for the example of a model linac loosely inspired by the electron injector Linac 2 for the positron intensity accumulator [14]. Table I gives the parameters of the model. Note that impedance and loss factor are specified with the convention power $=V^{2} / 2 R$. The target energy gain and beam current in the following figures are $50 \mathrm{MeV}$ and $100 \mathrm{~mA}$, respectively. Figure 2 shows the field along the linac as it fills, without beam loading. Figure 3 shows energy gain through the linac as a function of injection

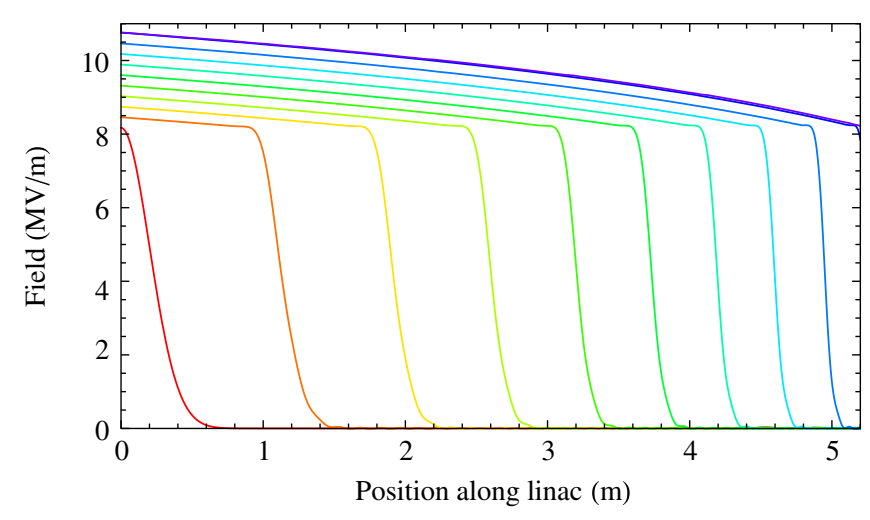

FIG. 2. Electric field along the length of the linac given in the table traced out at 100-ns intervals starting at $t=0$ (red). The rf drive is as shown in Fig. 4 tuned for 100-mA beam current. The leftmost (red) trace is the initial field profile at $t=0$, and the uppermost (blue) trace is the static field $\bar{E}_{z}(z)$. 


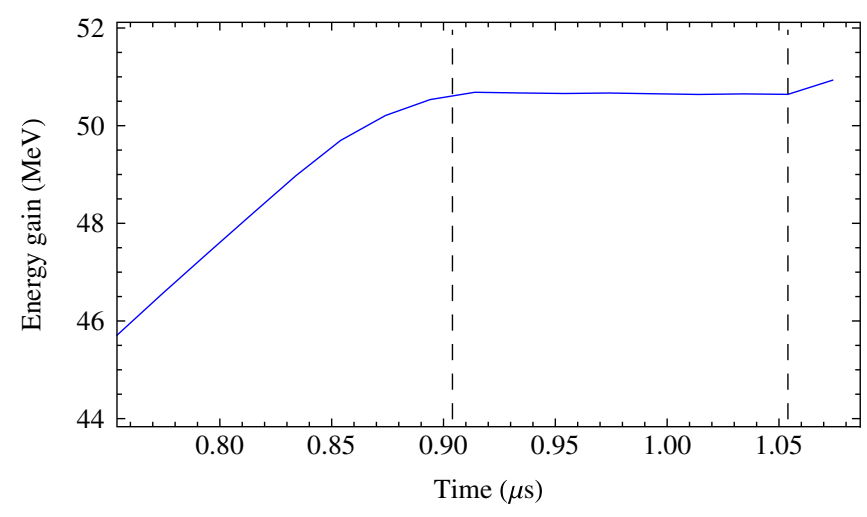

FIG. 3. Energy gain as a function of time through a 5.2-meter constant-gradient linac with beam and with constant rf drive. Injected current is $100 \mathrm{~mA}$. The dashed lines delimit beam injection.

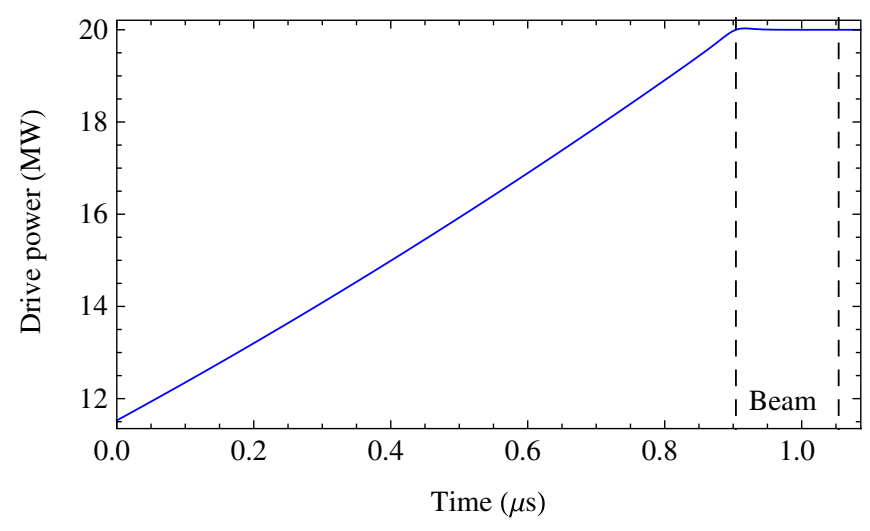

FIG. 4. The rf drive of the linac with beam that levels the energy gain as a function of time as shown in Fig. 3. Injected current is $100 \mathrm{~mA}$ and beam is on crest. The dashed lines delimit beam injection.

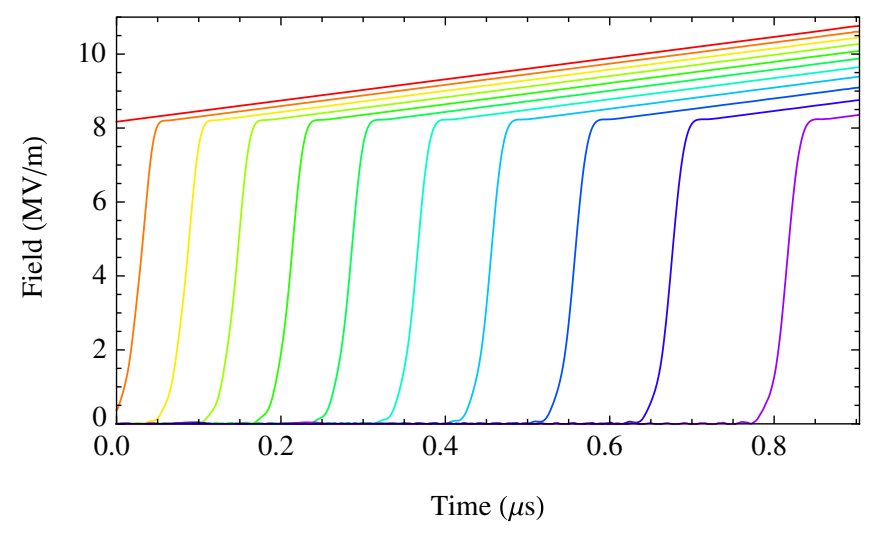

FIG. 5. Electric field as a function of time traced out at fixed positions along the linac. The uppermost (red) trace is at $z=0$ and successive traces are at $0.5-\mathrm{m}$ intervals ending at $5.0 \mathrm{~m}$ (violet). The rf drive is as shown in Fig. 4, tuned for 100-mA beam current. The uppermost (red) trace is the injected rf field $\hat{E}_{z}(0, t)$ showing the linear ramp. There is no beam injected during the time shown. time given the drive power shown in Fig. 4. Equation (26) was solved using the Mathematica [15] function NDSOLVE. Nonsharp boundary conditions were applied as a means to limit the computer memory demanded by this solver. Particle velocity is infinity (zero transit time). Figure 5 shows the electric field as a function of time at several positions along the linac's length, again as it fills.

\section{SUMMARY}

A time-dependent convective-transport equation for arbitrarily varying traveling-wave accelerating structures was applied to the problem of preparing an initial field profile in the structure that is static under beam loading, consequently providing constant energy gain throughout subsequent beam injection. Finite particle velocity (nonzero transit time) is handled. Closed-form expressions specific to constant-gradient linacs were derived with (a) varying loss factor and $Q$, (b) constant loss factor and varying $Q$, and (c) with loss factor and $Q$ both constant. Case (b) [and (c)] requires that linearly ramped rf amplitude be applied, at least where beam transit time is small. The transport equation was numerically solved for the last case to illustrate these results.

Although these results do not obviate the need for calculations for dispersion, nor for adaptive feedforward of the rf in real linacs, they do allow for more accurate estimates of power ramps and for better and more simply computed performance estimates of these machines.

\section{ACKNOWLEDGMENTS}

Thanks go to Hengjie Ma and Timur Shaftan for helpful comments. This work was performed under Contract No. 126615 for Brookhaven Science Associates, LLC and the NSLS-II project.

[1] R. Brinkmann, G. Materlik, J. Rossbach, and A. Wagner, Report No. DESY-97-048, Deutsches ElektronenSynchrotron, 1997.

[2] N. Holtkamp and A. Jostingmeier, Report No. DESY-96043, Deutsches Elektronen Synchrotron, 1996.

[3] J.E. Leiss, in Linear Accelerators, edited by P.M. Lapostolle and A.L. Septier (North-Holland Publishing Company, Amsterdam, 1969), Chap. B.1.3, pp. 147-172.

[4] C. Adolphsen et al., in Proceedings of the Particle Accelerator Conference, Vancouver, BC, Canada, 1997 (IEEE, Piscataway, NJ, 1998), p. 1676.

[5] K. U. Kasemir, M. Champion, M. T. Crofford, and H. Ma, in Proceedings of the Particle Accelerator Conference (PAC 05), Knoxville, Tennessee, 2005, edited by C. Horak (IEEE, Piscataway, NJ, 2005), p. 1467.

[6] E. L. Chu, Technical Note No. TN-62-069, Stanford Linear Accelerator Center, 1962. 
[7] R. B. Neal, Report No. 513, Stanford University Microwave Laboratory, 1958.

[8] R. M. Jones, V. A. Dolgashev, R. H. Miller, C. Adolphsen, and J. W. Wang, arXiv:physics/0307034.

[9] R. M. Jones, V. A. Dolgashev, and J. W. Wang, Phys. Rev. ST Accel. Beams 12, 051001 (2009).

[10] K. A. Thompson and R. D. Ruth, in 1993 Particle Accelerator Conference (PAC 93), Washington, DC, 1993, CEBAF (IEEE, Piscataway, NJ, 1993), p. 3693.

[11] E.L. Chu, Technical Note No. TN-63-009, Stanford Linear Accelerator Center, 1963.
[12] R. D. Ruth et al., Report No. SLAC-PUB-6293, Stanford Linear Accelerator Center, 1993.

[13] R. L. Bishop and S.I. Goldberg, Tensor Analysis on Manifolds (Dover Publications Inc., New York, 1980).

[14] J. Clendenin, L. Rinolfi, K. Takata, and D. J. Warner, Compendium of Scientific Linacs (CERN, Geneva, Switzerland, 1996), published in XVIII International Linac Conference.

[15] Mathematica Version 7 (Wolfram Research, Champaign, IL, 2008). 